\title{
Late-Onset Aspergillus Fumigatus Infection of an Aortic Stent Graft in an Immunocompetent Patient
}

\author{
Fatos Ballazhi ${ }^{1} \quad$ Michael Weyand ${ }^{1} \quad$ Werner Lang $^{2}$ Christoph Schoerner ${ }^{3}$ Timo Seitz $^{1}$ \\ ${ }^{1}$ Department of Cardiac Surgery, Friedrich-Alexander University, \\ Erlangen-Nuremberg, Germany \\ 2 Department of Vascular Surgery, Friedrich-Alexander University, \\ Erlangen-Nuremberg, Germany \\ ${ }^{3}$ Institute of Clinical Microbiology, Immunology and Hygiene, \\ Friedrich-Alexander University Erlangen-Nuremberg, \\ Erlangen, Germany \\ Thorac Cardiovasc Surg Rep 2015;4:56-58.
}

\begin{abstract}
Aspergillus fumigatus as a clinical entity is difficult to diagnose. We present a case, which could facilitate diagnosis and management of the aforementioned disease. A 60-yearold man with stent graft implantation in the descending aorta (6 years ago) presented with fever, night sweats, and weight loss over 5 months. Leukocytosis and elevated Creactive protein were constantly spiking. Blood cultures were negative. Notably, the serum immunoglobulin $\mathrm{E}(\mathrm{lgE})$ level was strongly elevated $(>1,000 \mathrm{U} / \mathrm{mL})$. Anamnestically, the patient suffered from a mild form of atopic dermatitis and bronchial asthma. The pulmonary status showed no abnormalities in the computed tomography image. Nonetheless, a chest scan revealed a suspected abscess around the stent graft of the descending aorta. Extra-anatomic ascending to descending aortic bypass (Gelsoft $22 \mathrm{~mm}$, Vascutek, Juchinnan, Scotland, United Kingdom) was performed. Intraoperative samples revealed $A$. fumigatus. These findings were confirmed by polymerase chain reaction analysis. Infection by $A$. fumigatus represents a diagnostic challenge because blood cultures are usually negative, but expeditious treatment is required to

Keywords

- graft infection

- aorta

- Aspergillus fumigatus prevent occurrence of irreversible complications. A late graft infection, possibly caused by $A$. fumigatus should be suspected in patients with implanted grafts, who suffer from unexplained, blood culture-negative fever that does not respond to antibiotics and who have a history of dermatitis or bronchial asthma with elevated IgE antibodies.
\end{abstract}

\section{Introduction}

Vascular stent infections caused by Aspergillus fumigatus have rarely been described. Invasive aspergillosis (IA) is the most devastating complication of Aspergillus-related diseases, targeting severely immunocompromised patients. ${ }^{1}$ Aspergillus vascular graft infections are serious complications of vascular surgical procedures and have been associated with high mortality despite aggressive medical and surgical interven-

received

January 12, 2015

accepted after revision

April 28, 2015

published online

July 10, 2015
DOI http://dx.doi.org/ 10.1055/s-0035-1555013 ISSN 2194-7635. tions. Aspergillosis as a clinical entity is difficult to diagnose, and is detected often only during microbiological investigations of intraoperative or postmortem tissue samples. ${ }^{2}$

\section{Case Report}

A 60-year-old man with a 6 year history of stent graft implantation (EndoFit 30/200, LeMaitre Vascular, Burlington, Massachusetts) in the descending aorta due to
License terms

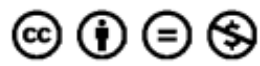

(c) 2015 Georg Thieme Verlag KG
Stuttgart · New York 
trauma-induced descending aneurysm (7 cm diameter) presented with fever, night sweats, and weight loss over 5 months. He was initially treated with antibiotics by a general practitioner, which proved unsuccessful. Stress electrocardiogram revealed signs of ischemia and led the patient to our clinic to exclude coronary artery diseases. Three-vessel coronary artery diseases with hemodynamic stenosis (left anterior descending [LAD] coronary artery, right circumflex [LCX], and right coronary artery [RCA]) and impaired left ventricle-function (ejection fraction [EF] 45\%) were found. The patient had leukocytosis and elevated CRP values. Despite intravenous antibiotic therapy, fever persisted. Anticoagulation therapy (phenprocoumon) was started due to paroxysmal atrial fibrillation which was probably triggered by infection. A few days after admission paresthesia of the left arm was noted and a cerebral hemorrhage was diagnosed (-Fig. 1A). A chest CT scan showed an attached thrombus, air bubbles, and a suspected abscess around the stent graft of the descending aorta (-Fig. 1B, C). Blood cultures remained negative despite an incubation period of 3 weeks. Notably, the serum immunoglobulin $\mathrm{E}(\mathrm{IgE})$ level was strongly elevated $(>1,000$ $\mathrm{U} / \mathrm{mL}$ ). Anamnestically, the patient suffered from a mild form of atopic dermatitis and bronchial asthma. An inhaled bronchodilator was used as needed. The pulmonary status showed no abnormalities in the CT image.
A single-photon emission computed tomography (SPECT) and a Positron emission tomography with 2-deoxy-2-[fluorine-18]fluoro-D-glucose with computed tomography (18F-FDG PET/CT) did not identify any infection and pathological structure throughout the body. However, a mild inflammatory reaction in the aneurysmal sac was detected (-Fig. 1D). Three coronary artery bypass grafting (CABG) and an extra-anatomic ascending-to-descending aortic bypass (Gelsoft $22 \mathrm{~mm}$, Vascutek, Juchinnan, Scotland, United Kingdom) were performed. -Fig. 1E shows the end-to-side anastomosis of the ascending aorta and CABG. A silver Dacron prosthesis was tunneled to the left pleural space and an end-to-end anastomosis of the graft prosthesis and the unaffected part of the descending aorta, near to the thoracoabdominal crossing, was performed. The aorta was ligated at the junction between aortic arch and descending aorta, close to the origin of the subclavian. The stent graft was removed in toto. Severe inflammation of the aneurysmal sac was noted. The infection process extended close to the trachea. A conspicuous whitish material was thoroughly debrided from the inflamed aneurysmal sac. Microscopical examination of intraoperative samples revealed fungal hyphae (Gram and stilbene stain). Cultures yielded the growth of colonies typical for $A$. fumigatus. These findings were confirmed by polymerase chain reaction analysis.

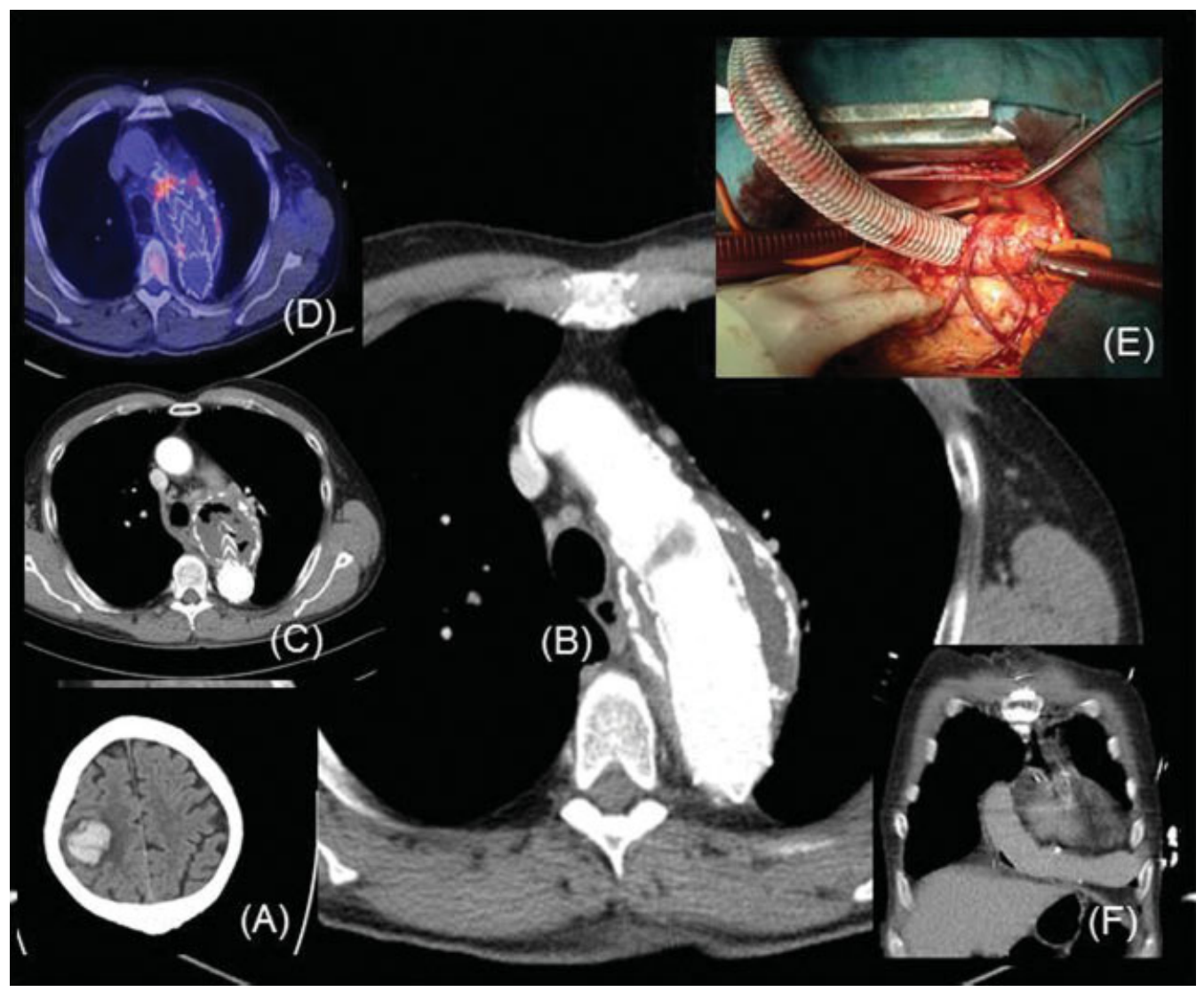

Fig. 1 (A) Cerebral hemorrhage, (B and C) abscess around the stent graft of the descending aorta with attached thrombus and air inclusions, (D) mild inflammatory reaction detected by SPECT imaging technique, (E) end-to-side anastomosis of ascending aorta and coronary artery bypass grafting, (F) the correct contrast filling of vascular graft. SPECT, single-photon emission computed tomography. 
At the postoperative day 1 , the patient was extubated. His response was adequate. On the 3rd postoperative day, the patient experienced respiratory failure and had to be reintubated. - Fig. 1F shows correct contrast-filling of the vascular graft. Several attempts of extubation failed. Respiratory exhaustion of the patient required peripheral venovenous extracorporeal membrane oxygenation (ECMO). Sputum-cultures were positive for enterococci. Chest-abdominal CT scan showed multiorgan microembolization. At postoperative day 10 , there was spontaneous bleeding from the thoracic drainage. A rethoracotomy revealed no bleeding source, but ubiquitous bleeding which could be tamponaded by stripe towels. Despite maximal therapy the patient died due to multiorgan failure and septic shock 11 days after operation. Postmortem pathology revealed primarily severe bacterial pneumonia as the cause of death.

\section{Discussion}

A. fumigatus is a ubiquitous fungus that can invade tissues and cause severe infections, primarily in immunocompromised individuals. Most often, this fungus is introduced via the respiratory tract. Vascular graft infections due to A. fumigatus have rarely been reported, even rare if the patient is immunocompetent. ${ }^{2,3}$

IA represents a diagnostic and clinical challenge because blood cultures remain almost always negative and aggressive treatment is required to prevent irreversible complications and lethal outcome. Excision of the infected prosthetic material and antifungal therapy should be performed rapidly.

Elevated serum IgE level are characteristic for allergic bronchopulmonary aspergillosis and atopic dermatitis. ${ }^{4,5}$ Immunological investigations in our patient revealed elevated IgE levels above 1,000 U/mL. Retrospectively, the asthmatic disease of our patient presumably facilitated the penetration and invasion of A. fumigatus. The aneurysmal sac infection was very close to the trachea which suggests that the infection spread via the trachea, although a true fistula was excluded by bronchoscopy. Interestingly, a left vocal cord paralysis was diagnosed months before the patient's admission.

From a diagnostic point of view, it is important to note that in our patient samples of bronchoalveolar lavages remained negative for $A$. fumigatus and did not contain elevated con- centrations of Aspergillus galactomannan antigen. In the serum, both the levels of anti-Aspergillus antibodies (indirect hemagglutination test [IHAT] 1:160) and of galactomannan antigen (index 1.0) were inconspicuous. These unexpected results are consistent with the interpretation that the fungal infection remained primarily localized between the aneurysm wall and the stent graft.

In conclusion, a fungal late-onset graft infection (e.g., with A. fumigatus) should be suspected in patients with implanted grafts, who suffer from unexplained, blood culture-negative fever that does not respond to antibiotics and who have a history of dermatitis or bronchial asthma with elevated IgE antibodies. Serological analyses for Aspergillus antibodies and galactomannan antigen should always be performed, although negative results never exclude an Aspergillus infection as illustrated by the present case. Expeditious antifungal treatment is required to prevent occurrence of irreversible complications.

\section{Note}

The article has been presented as a poster at: 30th Annual Meeting of the German Society of Vascular Surgery and Vascular Medicine; September 25, 2014; Hamburg, Germany.

\section{Acknowledgment}

The authors are grateful to Prof. Bogdan for editing and critical reading of the article.

\section{References}

1 Dagenais TR, Keller NP. Pathogenesis of Aspergillus fumigatus in Invasive Aspergillosis. Clin Microbiol Rev 2009;22(3):447-465

2 Rahkonen M, Hautala T, Syväniemi E, Takalo R, Kauma H. Latepresenting vascular graft infection caused by Aspergillus in an immunocompetent patient. Mycoses 2012;55(1):95-98

3 Calcaterra D, Bashir M, Gailey MP. Ascending aortic graft thrombosis and diffuse embolization from early endoluminal Aspergillus infection. Ann Thorac Surg 2012;94(4):1337-1339

4 Zmeili OS, Soubani AO. Pulmonary aspergillosis: a clinical update. QJM 2007;100(6):317-334

5 Leung DY, Boguniewicz M, Howell MD, Nomura I, Hamid QA. New insights into atopic dermatitis. J Clin Invest 2004;113(5):651-657 